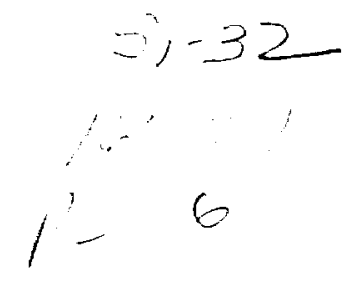

\title{
An Improved Linear Ion Trap Physics Package
}

\author{
J. D. Prestage \\ Communications Systems Research Section
}

\begin{abstract}
This article describes an improvement in the architecture of the physics package used in the Linear Ion Trap (LIT)-based frequency standard recently developed at JPL. This new design is based on the observation that ions can be moved along the axis of an LIT by applied dc voltages. The state selection and interrogation region can be separated from the more critical microwave resonance region where the multiplied local ascillator signal is compared with the stable atomic transition. This separation relaxes many of the design constraints of the present units. Improvements include increased frequency stability, and a substantial reduction in size, mass and cost of the final frequency standard.
\end{abstract}

\section{Introduction}

The primary reason that ions confined to electromagnetic traps are the basis for stable frequency standards is that in the containerless environment of a trap, processes that equalize atomic state populations and destroy coherence within the state-prepared atomic ensemble are very weak. In past work coherence times have been measured in an ensemble of trapped $\mathrm{Hg}^{+}$ions of over $30 \mathrm{sec}$ on the $40.5-\mathrm{GHz}$ transition. Such weak relaxation has permitted a resonance line width $\Delta \nu$ as small as $17 \mathrm{mHz}$ on the 40.5 $\mathrm{GHz}$ transition. This line-Q $\left(\nu / \Delta \nu=2 \times 10^{12}\right)$ is the highest ever measured on a microwave atomic transition $[1,2]$.

One disadvantage of a trapped ion-based frequency standard is the relatively low density of the confined ions. This leads to a low signal-to-noise ratio (SNR) in the detected atomic resonance and consequently limits clock stability. This situation was greatly improved by the use of the linear ion trap [2] where the number of trapped ions was increased by more than 10 over conventional hyperbolic traps. The stability reached with the improved SNR of the Linear Ion Trap (LIT) together with the high line$\mathrm{Q}$ has led to a tenfold improvement in clock stability over conventional ion-based clocks. Indeed, the present LITbased clock shows stability competitive with the best $\mathrm{H}$ masers for averaging times less than 10,000 sec and exceeds H-maser stability beyond $10,000 \mathrm{sec}$, making it the most stable of all clocks for long-term stability. The LIT clock is, however, relatively recent in its development and its configuration continues to evolve. The purpose of this article is to propose an improvement in the architecture of the current physics package which will lead to improved longterm stability, and a substantial reduction in size, mass and cost of the final frequency standard.

\section{Current LIT Operation}

The present form of the LIT physics unit for frequency standard operation is shown in Fig. 1. Ions are created inside the linear trap by an electron pulse which ionizes a weak vapor of parent neutral atoms introduced into the vacuum system from a heated $\mathrm{HgO}$ isotopic source. They are held in the four-bar linear trap shown inside the region 
enclosed by magnetic shields [3]. Before the stable atomic frequency of the trapped ions can be compared with the multiplied output of a local oscillator (LO) or flywheel oscillator, a population difference between the hyperfine levels of the ground state must be created. This is done via optical pumping with ultraviolet (UV) light from a laser or a discharge lamp. As shown in Fig. 1, optical pumping is carried out by a ${ }^{202} \mathrm{Hg}$ lamp whose output light is collected and focused onto the central portion of the ion cloud.

After state preparation, microwave radiation derived from the LO is passed onto the ions to make a frequency comparison with the stable atomic resonance. During this period it is crucial that the atomic resonance frequency not be perturbed by any changes in the trap environment. Such fluctuations will be transferred to the servoed LO, thereby degrading clock stability. Because the state selecting light will shift the atomic clock resonance, it is switched off during the microwave interrogation time. Following the microwave pulse, the lamp is turned on again to determine whether the microwave radiation has changed the population of the hyperfine levels of the atomic ion. Any frequency detuning of the LO from the reference atomic frequency will change the fluorescent light intensity measured when the lamp is turned on. These fluorescence changes are converted to a voltage and fed back to the frequency control port of the LO to keep it on frequency with the atomic oscillator.

It is apparent that the trap region is operated in two modes in the process of steering an LO. In the first mode, the atomic ions are prepared for microwave frequency comparison with the LO. In the second mode, the atomic frequency is compared with the multiplied output of the LO. During the state preparation and interrogation mode, there are no stringent requirements on environmental isolation or regulation, whereas during the resonance mode, great care must be exercised in regulation of the atomic environment to insure stable operation.

\section{Improved LIT Design}

Figure 2 shows a configuration of an ion trap-based clock where these modes of operation are carried out in two separate regions. The trap shown is a lengthened version of the LIT of Fig. 1. In the new architecture of Fig. 2 the region labeled ion loading and fluorescence is somewhat smaller in size than the trap shown in Fig. 1. A second linear trap has been added for a microwave resonance region.

A $\mathrm{dc}$ break forms a junction between these two regions to allow ions to pass from one region to another. The rf trapping voltage for transverse containment of the ions is continuous across this dc break. When the dc voltage levels of all four trap rods are the same in both the upper and lower regions, thermal motion of the ions will carry them through the junction with no change in axial velocity. When the four trap rods in the upper region are at negative dc voltage with respect to the lower region trap rods, ions within a trap radius or so of the junction will be transported across the junction into the upper region. Only the ions near the junction will experience the electric field forcing them across the gap. Since each ion is in thermal motion along the axis of the trap, it will reach the junction within a trap-length transit time (typically about $1 \mathrm{msec}$ ) and then be pulled into the upper region, emptying the lower region of ions. Similarly, when the upper region is dc biased positive with respect to the resonance region, all ions will be transported from the fluorescence region to the ion resonance region. The dc potential along the length of the trap that controls the region the ions occupy is shown in Fig. 3.

The separation of the clock resonance region from the optical and ion loading region relaxes many of the design constraints of the present units. The microwave resonance region can now be designed with no consideration of optical issues. A simple set of cylindrical shields and a solenoid supply a very uniform and stable magnetic environment. In Fig. 2 it is seen that the volume of the resonance region is now reduced by about 100 as the magnetic shields are 10 times smaller in diameter. Since the resonance region requires the most stringent temperature regulation of the entire physics package, the size reduction enables simplification of thermal control of the standard.

Similarly, the state selection optical design is now done with no worry of perturbing the atomic resonance. In practice, this means that the optical components are no longer required to be nonmagnetic as in the previous design. The custom-made copper-titanium flanged UV transmitting windows can be replaced with S-steel flanged windows, which are each about $\$ 2,000$ less expensive. The $\$ 4,000$ nonmagnetic UV light-collecting horn is replaced by an S-steel elbow connecting the trap region to the vacuum pumps. The light source can be moved much closer to the ion cloud, raising the possibility of using a collimated UV source with no collection mirror, thus further reducing the final package size. Similar modifications could be made in the collection arms to reduce size. These design changes would not allow stable clock operation if the ions underwent microwave clock resonances inside the optical state selection region as in the previous design, because of the large magnetic shifts of the atomic levels induced by the close proximity of the light source and detectors. 
Another simplification gained by relaxing the nonmagnetic requirement is in the electron gun assembly and its heater current supply. The electron source for ion creation is now remote from the atomic resonance region, allowing use of the standard (magnetic) filament base and a floatable $\mathrm{dc}$ current drive. In the design of Fig. 1, ions are created inside the resonance region and require a floating audio frequency filament heater current to prevent the residual dc magnetic field from the electron gun from shifting the atomic ion frequency.

Additionally, the largest frequency offset and potential instability can be reduced greatly by selecting a somewhat longer trapping length in the atomic resonance region. This frequency shift stems from the finite (i.e., nonzero) ion cloud diameter in which ions spend time in regions of large rf trapping fields where their motion results in frequency pulling via the second-order Doppler or relativistic time dilation effect. The magnitude of this offset depends only on the linear ion density, $N / L$, and is given by [3]

$$
\left(\frac{\Delta f}{f}\right)=-\left(\frac{e^{2}}{8 \pi \epsilon_{0} m c^{2}}\right) \frac{N}{L}
$$

An increase in the resonance trapping length to about $200 \mathrm{~mm}$ would reduce the clock sensitivity to ion number variations by about a factor of 4 from that in the present system of Fig. 1. Since ion number stabilization to the 0.1percent level has been demonstrated for $10^{-15}$ frequency standard operation in that system, an ion number-induced instability noise floor below $2.5 \times 10^{-16}$ should be achievable in this modified configuration.

\section{Conclusions}

An improved architecture for a linear ion trap-based frequency standard is described. Its design is a natural extension of the original LIT and separates the state selection and ion creation region from the atomic resonance region. Many of the design constraints of the present configuration are eliminated and a smaller, cheaper, more stable frequency standard becomes possible.

\section{References}

[1] J. D. Prestage, R. L. Tjoelker, G. J. Dick, and L. Maleki, "Ultra-Stable $\mathrm{Hg}^{+}$ Trapped Ion Frequency Standard," Journal of Modern Optics, vol. 39, pp. 221$232,1992$.

[2] J. D. Prestage, R. L. Tjoelker, R. T. Wang, G. J. Dick, and L. Maleki, " $\mathrm{Hg}^{+}$ Trapped Ion Standard Performance With the Superconducting Cavity Maser Oscillator as L.O.," Proc. 1992 IEEE Frequency Control Symposium, pp. 58-63, 1992.

[3] J. D. Prestage, G. J. Dick, and L. Maleki, "New Ion Trap for Frequency Standard Applications," Journal of Applied Physics, vol. 66, pp. 1013-1017, 1989. 


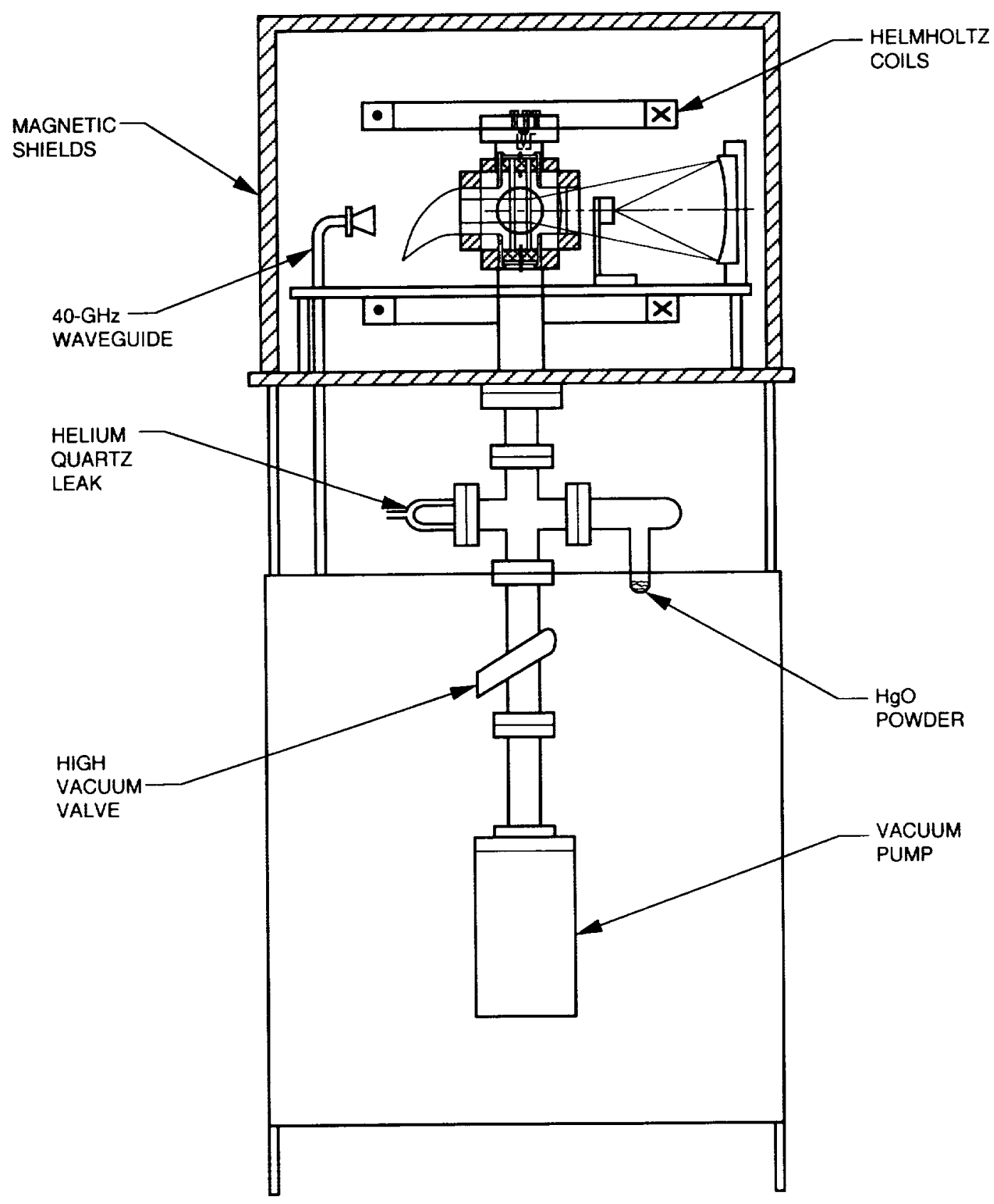

Fig. 1. Present physlcs unlt for the linear ion trap-based frequency standard. $\mathrm{Hg}$ lons are state prepared and interrogated with the multiplled output of a local osclilator in the lon trap housed inside the magnetlc shlelded region. The apparatus 18 about $2 \mathrm{~m}$ high by $1 \mathrm{~m}$ on a side. 


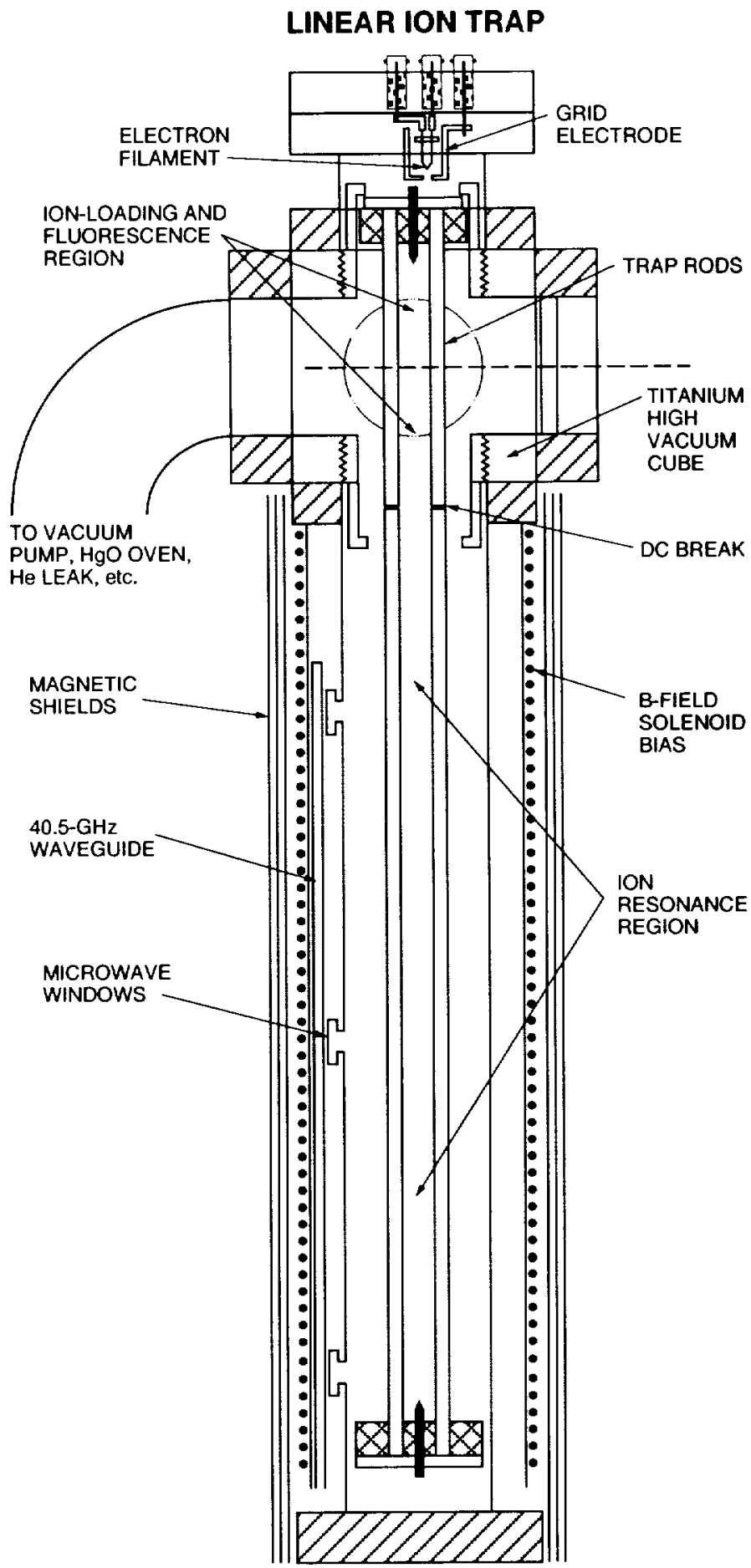

Fig. 2. Improved physics unit for the trapped lon frequency standard. $\mathrm{Hg}$ lons are created and state prepared in the upper region of the trap, then electrically moved Into the lower reglon, where the $\mathrm{Hg}^{+}$atomic resonance frequency is compared with the local oscillator frequency. The lons are then moved back to the state selection region to check for frequency detuning trom the LO during the resonance comparison. Overall dimensions are about $0.5 \mathrm{~m}$ by $10 \mathrm{~cm}$. 
(a)

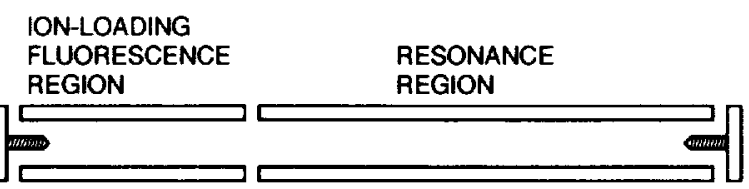

(b)

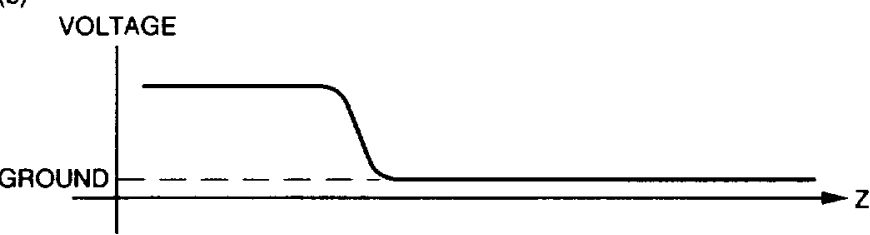

(c)

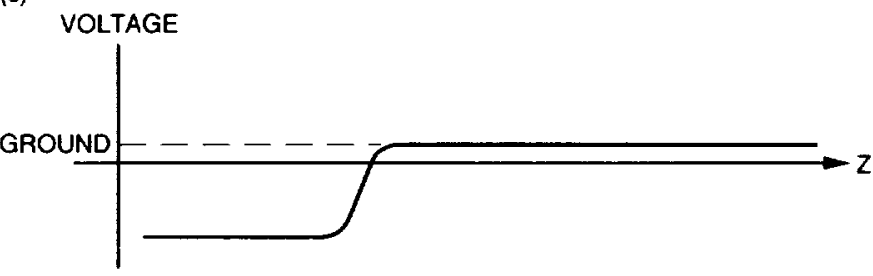

Fig. 3. The improved linear ion trap with separated state selectlon and microwave resonance reglons: (a) the complete lon trap, showing the dc Junction; (b) a positlve dc blas is applied to the loading and Interrogation trap rods forcing the lons into the resonance region; and (c) lons are drawn back into the fluorescence reglon by applylng a negative blas. 